\title{
NOTES
}

\section{Structural Insights from the Extended X-Ray Absorption Fine Structure of Polyacrylates with Paramagnetic Tetraphenylporphynato-Silver(II) and -Erbium(III) Derivatives}

\author{
Atsushi KaJiwara, Mikiharu KamaCHI, ${ }^{\dagger}$ and Hironobu MaEdA* \\ Department of Macromolecular Science, Faculty of Science, \\ Osaka University, Toyonaka, Osaka 560, Japan \\ * Department of Chemistry, Faculty of Science, Okayama University, \\ 3-1-1 Tsushima-Naka, Okayama 700, Japan
}

(Received September 17, 1990)

\begin{abstract}
KEY WORDS EXAFS / Polyacryloyloxytetraphenylporphynatosilver" / Polyacryloyloxytetraphenylporphynato-erbium ${ }^{\mathrm{III}} \mathrm{OH}$ / Propyloyloxytetraphenylprophynatosilver ${ }^{\mathrm{II}}$ / Tetraphenylporphynato-erbium ${ }^{\mathrm{III}} \mathrm{OH}$
\end{abstract}

In the course of study on magnetic behavior of polymers containing paramagnetic species, we found that poly(5-(4-acryloyloxyphenyl)10,15,20-triphenylporphynatosilver(II) (polyAOTPPAg ${ }^{\text {II }}$, Figure $1, M=A g^{\text {II }}$ ) indicated the occurrence of antiferromagnetic interaction between TPPAgII moieties in the side chains, although tetraphenylporphynatosilver ${ }^{\text {II }}$ (TPPAg $\left.{ }^{\mathrm{II}}\right)$ and 5-(4-propyloyloxyphenyl)10,15,20-triphenylporphynatosilver ${ }^{\text {II }}$ (POTP$\mathrm{PAg}^{\text {II }}$ ) showed only a weak antiferromagnetic

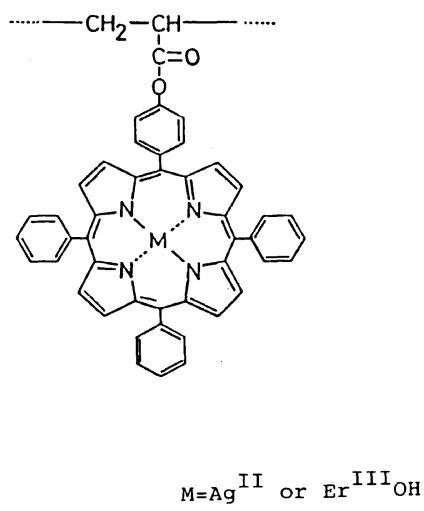

Figure 1. PolyAOTPP-AgII, or $\mathrm{Er}^{\mathrm{III}} \mathrm{OH}$.

\footnotetext{
† To whom correspondence should be addressed.
}

interaction. ${ }^{1,2}$ Comparison of visible spectra of polyAOTPPAgI and TPPAg ${ }^{\text {II }}$ suggested the possibility that TPPAgII moieties bound to a polymer chain are forced to make some electronic interaction due to their close approach. $^{1,2}$ Similarly, poly((5-(4-acryloyloxyphenyl)-10,15,20-triphenylporphynato)erbium ${ }^{\text {III }}$-hydroxide) (polyAOTPPEr ${ }^{\text {III }} \mathrm{OH}$ ) showed ferromagnetic behavior in the low temperature, while TPPEr ${ }^{\mathrm{III}}$ was paramagnetic in the same temperature range. ${ }^{3,4}$

Since these polymers were amorphous, no structural information concerning the origin of polymer effect on magnetic behavior was obtained by X-ray diffraction method. It is well known that EXAFS measurements provide specific information about the environment of the absorbing atoms not only in crystalline compounds but also in amorphous compounds. Accordingly, the EXAFS spectroscopy is quite suitable for the structural information on the origin of the polymer effect on the magnetic behavior.

EXAFS measurements were performed by using the synchrotron radiation from the Photon Factory (PF) at the National Laboratory of High Energy Physics (KEK, Tsuku- 
ba). ${ }^{5)}$ The silver $\mathrm{K}$-edge and erbium $\mathrm{L}_{\mathrm{III}}{ }^{-}$ edge X-ray absorption spectra were taken with the transmission mode at temperatures between $20 \mathrm{~K}$ and room temperature, using a
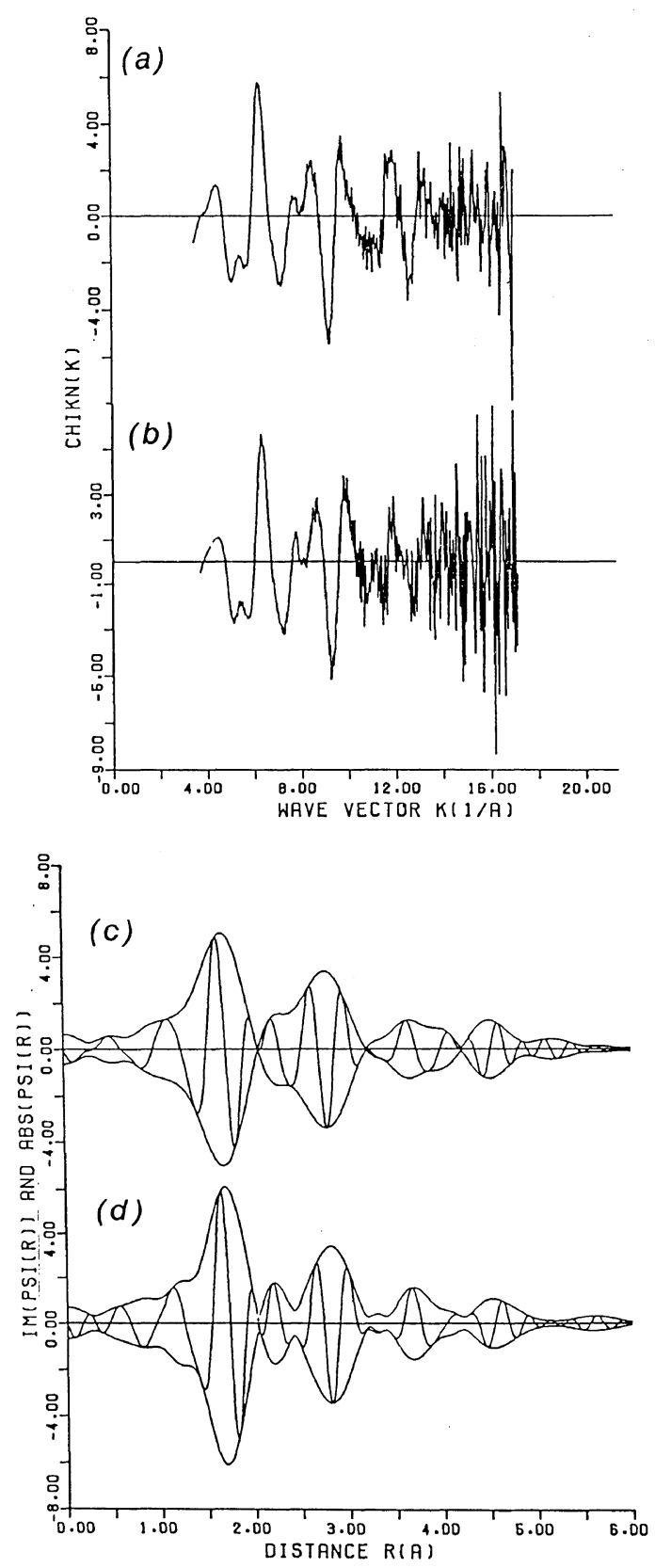

Figure 2. Normalized $X(k)$ vs. $k$ plot ((a) and (b)) and Fourier transform ((c) and (d)) for polyAOTPPAgII and POTPPAg", respectively. beam line BL-10B with a channel-cut silicon(300) crystal monochromator under a ring operating condition of $2.5 \mathrm{GeV}$ and $200 \mathrm{~mA}$ maximal current. The energy resolu-
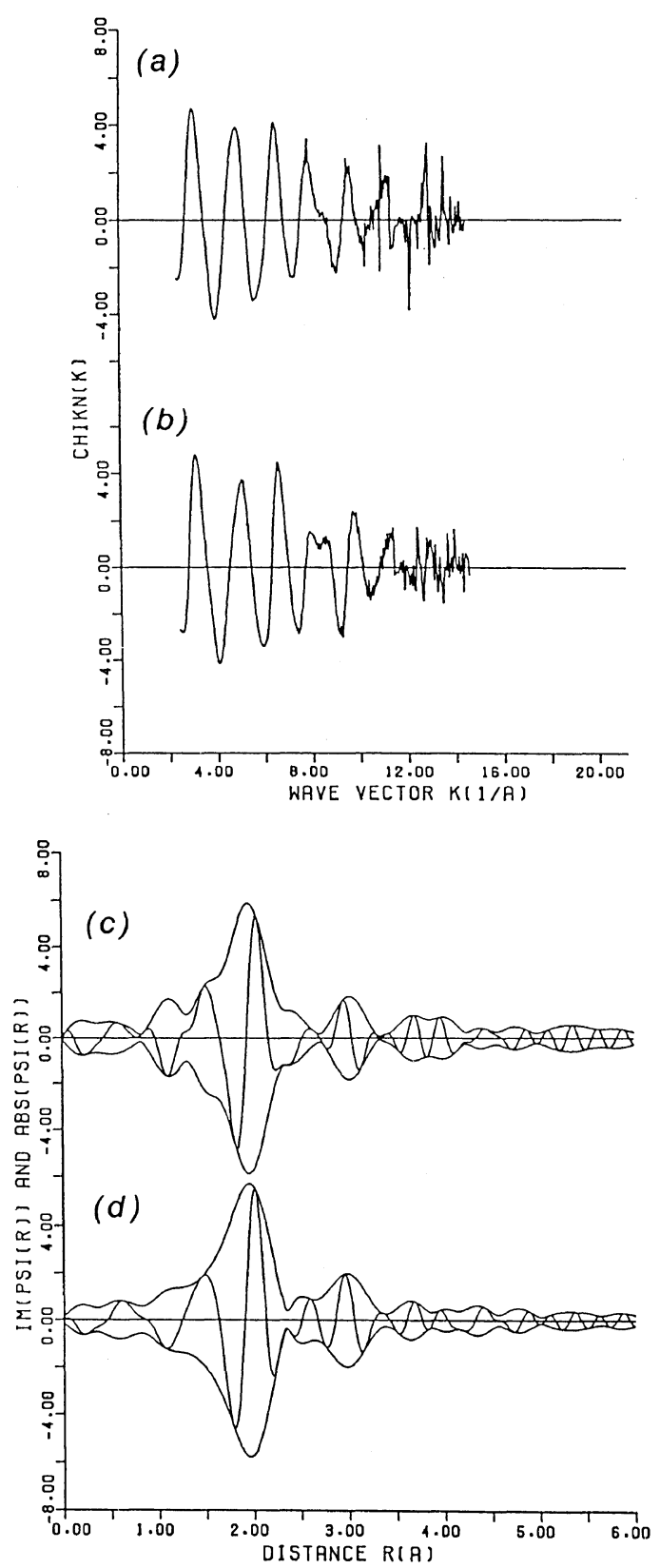

Figure 3. Normalized $X(k) v s . k$ plot ((a) and (b)) and Fourier transform ((c) and (d)) for polyAOTPPEr ${ }^{\mathrm{III} O H}$ and TPPEr ${ }^{\mathrm{III}} \mathrm{OH}$, respectively. 
tion is estimated to be about $0.5-1.0 \mathrm{eV}$ in the present study.

The porphyrin polymers ${ }^{1,4}$ were ground to fine powder, mixed with polyethylene powder, and then molded to disk with high pressure. A closed cycle cryostat was used for the low temperature measurements. The sample was cooled by thermal conduction method. The interference function $\chi(k)$ was extracted from each data set using standard techniques. ${ }^{6}$

The EXAFS oscillations, $k^{3} \chi(k) v s$. $k$ (in $\AA^{-1}$ ), of polyAOTPPAg ${ }^{I I}$ and the POTPPAg ${ }^{\text {II }}$ are shown in Figures 2(a) and 2(b), respectively. The Fourier transforms of these oscillations show peaks corresponding to the atomic positions around the X-ray absorbing atoms, where the peak positions are shifted a few tenths of an angstrom from the actual interatomic distances because of the EXAFS phase shift (Figures 2(c) and 2(d)).

Figures 2(c) and 2(d) show the Fouriertransformed curves for the silver K-edge, where the labels indicate only the dominant contributions calculated from the crystal structure. ${ }^{7}$ The first peak consists of four $\mathrm{Ag}-\mathrm{N}$ pairs $(\sim 1.70 \AA)$. The next peak is due to the second-nearest-neighbor environment, consisting of eight carbon atoms $(\sim 2.75 \AA)$.

In order to determine the structural parameters, we used the nonlinear least-squares fitting to the filtered data with a multiple-term semiempirical expression of the EXAFS formula. $^{8,9}$ Both the Ag-N pair $(0.67<r<$ $2.11 \AA)$ and Ag-C pair $(2.28<r<3.90 \AA)$ were analyzed by the single-shell fit using Teo and Lee's ${ }^{10}$ phase shifts and back-scattering amplitudes.

The numerical results are summarized in Table I. Table I shows that structural parameters of polyAOTPPAgII are almost the same as those of POTPPAgII. Therefore, it is concluded that the bond distances of $\mathrm{Ag}-\mathrm{N}$ and $\mathrm{Ag}-\mathrm{C}$ are not influenced by being bound to a polymer chain, although an electronic interaction takes place.

Figures 3(a) and 3(b) show normalized EXAFS spectra of polyAOTPPEr ${ }^{\mathrm{III}} \mathrm{OH}$ and TPPEr ${ }^{\mathrm{III}} \mathrm{OH}$ at room temperature. The Fourier transforms of these spectra provided the radial structure functions as shown in Figures 3(c) and 3(d). These Figures exhibit two distinct radial structure peaks at about 1.90 and $2.90 \AA$, for both polyAOTPPEr ${ }^{\mathrm{III}} \mathrm{OH}$ and TPPEr ${ }^{\mathrm{III}} \mathrm{OH}$, respectively. Their structural parameters were determined by a curve-fitting analysis to their Fourier-filtered EXAFS spectra. The best least-square analysis was obtained by using four nitrogens, oxygen, and eight carbons. The structural parameters obtained by the best fit are shown in Table I. Accordingly, two radial structure peaks in Figures 3(c) and 3(d) are ascribed to back scattering from the neighboring nitrogen atoms, oxygen atom, and eight carbon atoms. Weaker peaks were observed in

Table I. Best fit least-squares refined interatomic distance, Debye-Waller factor $(\sigma)$, scale factor $(B)$, and coordination number $(N)$ at $300 \mathrm{~K}$

\begin{tabular}{ccccccc}
\hline \multicolumn{1}{c}{ Sample } & Shell & \multirow{2}{*}{$E_{0} / \mathrm{eV}$} & $R / \AA$ & $\sigma / \AA$ & $B$ & $N$ \\
\hline POTPPAg(II) & Ag-N & 25502 & $2.092(0)$ & $0.055(0)$ & $0.603(1)$ & 4 \\
& Ag-C & & $3.114(0)$ & $0.070(0)$ & $0.387(0)$ & 8 \\
polyAOTPPAg (II) & Ag-N & 25496 & $2.084(4)$ & $0.047(3)$ & $0.603(0)$ & 4 \\
\multirow{2}{*}{ TPPEr(III)OH } & Ag-C & & $3.124(1)$ & $0.050(0)$ & $0.377(3)$ & 8 \\
& Er-N & 8356 & $2.356(3)$ & $0.083(2)$ & $1.008(0)$ & 4 \\
polyTPPEr(III)OH & Er-O & & $2.945(1)$ & $0.060(1)$ & $0.500(0)$ & 1 \\
& Er-C & & $3.320(0)$ & $0.082(0)$ & $0.500(0)$ & 8 \\
& Er-N & 8356 & $2.395(1)$ & $0.086(1)$ & $1.008(0)$ & 4 \\
& Er-O & & $2.942(16)$ & $0.053(18)$ & $0.500(0)$ & 1 \\
& Er-C & & $3.320(9)$ & $0.067(6)$ & $0.500(0)$ & 8 \\
\hline
\end{tabular}


the longer radial distance, but their intensities were not strong enough to be distinguishable from the ghost peaks.

From the present EXAFS data, it has not been possible to determine the metal-metal distance in the polymers. But, the unchanged structure around the metal ion at various temperatures indicates that the possibility of the appearance of magnetic interaction through the characteristic properties of polymers, e.g., successive, covalent bonding of paramagnetic species.

New information was obtained on the tetraphenylporphynato rare-earth ions. Only a few data due to $\mathrm{X}$-ray analysis has been reported on the molecular structure of tetraphenylporphynato rare-earth ion. ${ }^{11}$ To our knowledge, no data has been reported on the molecular structure of the TPPEr ${ }^{\mathrm{III} X}$ ( $\mathrm{X}=$ counter anion). Table I shows that the Er-N distance of TPPEr ${ }^{\mathrm{III}} \mathrm{OH}$ is longer than the Ag-N distance of TPPAg ${ }^{\text {II }}$ whose Ag ion has been reported to locate in the center of the porphyrin rings. Er ${ }^{\mathrm{III}}$ ion was located $1.09 \AA$ apart from the center of the porphyrin ring for monomeric species. The Er-N distance of polymer is longer than that of monomeric complex by about $0.04 \AA$. This difference is meaningful and seems to be concerned with the difference in electronic structure of porphyrin rings between polymer and monomer, because less extinction coefficients have been observed in the Soret band of the former as compared with that of the latter. ${ }^{3}$

Acknowledgments. This work was partly supported by a Grant-in-Aid for Scientific Research of Priority Areas of "Macromolecular Complexes" (No. 63612001) from the Ministry of Education, Science, and Culture of Japan.

\section{REFERENCES}

1. M. Kamachi, H. Akimoto, W. Mori, and M. Kishita, Polym. J., 16, 23 (1986).

2. S. Nozakura and M. Kamachi, Makromol. Chem. Suppl., 12, 255 (1985).

3. M. Kamachi, X. S. Cheng, H. Aota, W. Mori, and M. Kishita, to be published.

4. M. Kamachi, X. S. Cheng, S. Nozakura, Abstracts of Papers, the 5th Symposium in Rare Earths, Tokyo, 1987, p 180.

5. H. Oyanagi, T. Matsushita, M. Ito, and H. Kuroda, KEK Report, 80-83 (1984).

6. H. Maeda, J. Phys. Soc. Jpn., 56, 2777 (1987).

7. W. R. Scheidt, J. U. Mondal, C. W. Eigenbrot, A. Adler, and L. J. Radonovich, Inorg. Chem., 25, 795 (1986).

8. E. A. Stern, Phys. Rev., B10, 3027 (1974).

9. B. K. Teo, M. R. Antonio and B. A. Averrill, J. Am. Chem. Soc., 105, 3751 (1983).

10. B. K. Teo and P. A. Lee, J. Am. Chem. Soc., 101, 2815 (1979).

11. A. Dormond, B. Belkalem, P. Charpinm, M. Lance, D. Vigner, G. Folcher, and R. Guilard, Inorg. Chem., 25, 4785 (1986). 